\title{
COPERNICUS - PRACTICE OF DAILY LIFE IN A NATIONAL MAPPING AGENCY?
}

\author{
T. Wiatr, G. Suresh, R. Gehrke, M. Hovenbitzer* \\ Federal Agency for Cartography and Geodesy (BKG), Richard-Strauss-Allee 11, 60598 Frankfurt am Main, Germany \\ (thomas.wiatr, gopika.suresh, ralf.gehrke, michael.hovenbitzer)@bkg.bund.de
}

SpS9: GALILEO and COPERNICUS

KEY WORDS: Copernicus programme, Copernicus services, land monitoring, public authorities, BKG projects

\begin{abstract}
:
Copernicus is an European system created for Earth observation and monitoring. It consists of a set of Earth observation satellites and in-situ sensors that provide geo-information that are used, through a set of Copernicus services, for applications related to the environment and global security. The main services of the Copernicus programme address six thematic areas: land, marine, atmosphere, climate change, emergency management and security. In Germany, there is a national service team of Copernicus service coordinators, who are responsible for the national development of the Copernicus services and for providing user-specific information about the Copernicus processes. These coordinators represent the contact points for all the programmes and services concerning their respective Copernicus theme. To publish information about Copernicus, national conferences and workshops are organised. Many people are involved in planning the continuous process of bringing the information to public authorities, research institutes and commercial companies. The Federal Agency for Cartography and Geodesy (Bundesamt für Kartographie und Geodäsie, BKG) is one such organisation, and is mainly responsible for the national land monitoring service of Copernicus. To make use of the freely available data from the Copernicus programme, the Federal Agency for Cartography and Geodesy is currently developing new applications and projects in the field of remote sensing and land monitoring. These projects can be used by other public authorities as examples on how to use the Copernicus data and services for their individual demands and requirements. Copernicus data and services are currently not very commonly used in the daily routine of the national mapping agencies, but they will soon be.
\end{abstract}

\section{INTRODUCTION}

\subsection{The Copernicus Programme}

The Copernicus programme, coordinated and managed by the European Commission, has two main components which are related to each other; namely i) the Copernicus space component and ii) the Copernicus services with the in-situ component. The space component includes a connected structure of different organisations (e.g. ESA, EUMETSAT) and ground segment operations conducting the data postprocessing and evaluation, as well as the data collection from six different Sentinel satellite missions (Figure 1). Currently the first Sentinel- 1, 2, 3 missions have been launched and the other complementary satellites $(4,5,6)$ are planned to be launched until 2020 (www.esa.int). In addition, a second satellite for all Sentinel missions are planned to be launched after 2020.

Every satellite mission has individual scopes and different recording systems for acquiring the remotely sensed data. Therefore, the space components produce a huge amount (big data) of different datasets (with various resolutions, footprints, and produced by using active and passive sensors, etc.) for different customers with various applications. These userspecific datasets and individual applications are associated with the Copernicus services. The Copernicus services are divided into six thematic areas: land, marine, atmosphere, climate change, emergency management and security. These different services provide a means to support a wide range of interdisciplinary subjects and have the potential to find solutions for concrete application problems. One such application, and the focus of this paper, is the land monitoring service of Copernicus (Figure 1).

The land monitoring service provides data, from the local to the global scale, regarding information about land cover and land use. On a global scale, several bio-geophysical variables in mid and low resolution addressing the thematic areas vegetation, water cycle and energy budget are supplied. On the European scale, high resolution image mosaics, thematic raster and vector datasets (High Resolution Layers - HRL) are provided. These include the Corine Land Cover classification (CLC) starting from 1990, with the latest CLC for the year 2012, the CLC land cover changes over the same time period, and HRLs corresponding with the main themes from CLC: imperviousness, forests, grasslands, wetlands and permanent water bodies. At a local scale, more detailed and specific vector data at hot spots like urban areas, riparian zones or natural protected areas are provided. These datasets have a higher spatial and thematic resolution compared to the CLC. In the insitu data section, 3D data based on SRTM and ASTER data, and the LUCAS survey carried out by Eurostat, are provided. The access to the data is free for everyone (land.copernicus.eu). As with the land monitoring service data, the raw data from Sentinel-1, Sentinel-2 (and later on Sentinel-3) are provided free of charge to everyone by accessing the datahubs of the ESA (https://scihub.copernicus.eu/). The data are available through a web browser or by scripted access via an API hub and the access is limited to only two simultaneous downloads. Some of the data are not supplied in common data formats like geo-tiff (e.g. Sentinel-2 images), so an open source data viewing and conversion programme like the SNAP Toolbox (SNAP - ESA 
Sentinel Application Platform v2.0.2, http://step.esa.int), provided freely by ESA can be used.

The philosophy of the Copernicus programme, which is to make the data freely available, triggers the need and possibility for the development of many applications that use the provided data and services. Hence, this easy data access has a high probability to create new applications and, furthermore, participants have the option to use the Copernicus products for their own requirements. The fact that the user accessibility and download conditions have low restrictions allows equal opportunities for public, private and research institutions to enjoy the social and economic benefits of such an initiative. Consequently, it provides a platform to establish synergy at a national level, as well as fulfil the requirements of international stakeholders, policymakers and public authorities (Jetzek, 2013). Furthermore, the use of the data and products provided by the Copernicus programme have benefits not only for research in individual subjects, such as environment, agriculture, regional and local planning, forestry, statistic or hydrology, but provides a means to increase research between the various interdisciplinary subjects.

The availability of data from the same satellite and with the same specifications for the entire period of the satellite mission (7-10 years) provides another advantage to the Copernicus programme and allows reliable planning for the development of future products. This is especially important to improve and optimise the methods and processes used or workflows employed. A large temporal dataset also allows for the monitoring and evaluation of phenomena that only vary over longer periods in time.

\subsection{The national coordination of the Copernicus programme in Germany}

At a national level in Germany, the comprehensive European Copernicus programme is accompanied by the assignment of technical coordinators for every individual Copernicus service. They are appointed by the federal government for the national coordination of the Copernicus topics and have the following duties and responsibilities:

i) Coordination of the technical aspects of the Copernicus services and supporting the development of the services on behalf of the federal government.

ii) Spreading awareness and information about the Copernicus services and data among users in Germany. The users include authorities at all hierarchical levels as well as commercial users. iii) Asserting themselves as the main contact point for each appointed theme.

iv) Representation of the Federal Republic of Germany in the user forum of the European commission.

In particular, the Federal Agency for Cartography and Geodesy (Bundesamt für Kartographie und Geodäsie, BKG) in cooperation with the Federal Environment Agency of Germany (Umweltbundesamt UBA) is responsible for the land monitoring services of the Copernicus programme in Germany. The solution to assign technical coordinators for every individual Copernicus service is a special one in Europe. Thanks to this system, an efficient flow of information between the European commission and the user in Germany can be guaranteed. The technical coordinators represent the community of users in each individual technical theme and are responsible for spreading information about Copernicus within their communities.

\section{PROJECTS}

Land monitoring documents the changes in the conditions of an investigated region on the Earth's surface. Temporal changes in the environment can be detected and observed using multitemporal approaches. This provides periodic and consistent means to analyse the varying characteristics of the different surfaces and vegetation in an explored area. To accomplish this on a large scale, in high resolution and temporal variability, remote sensing products are frequently used. One topic in land monitoring is the analysis of the environmental changes in terms of land cover (LC) and land use (LU). This is important for biomass calculations, agricultural planning or urban city planning, etc. It is also important for disaster management and to establish fast rescue missions in cases where natural calamities like floods or earthquakes occur.

Since the Copernicus land monitoring services for Germany are handled by the BKG, several projects dealing with the evaluation and processing of Copernicus data are currently underway. These projects depend on the capability of the technical coordination or project leader to establish and promote the use of the Copernicus programme and products to other national and state level authorities and to demonstrate the capabilities and possibilities of the Copernicus programme. The current BKG projects comprise land applications, mainly, land cover and land use changes, and the resultant products support national and international demands from various administrations. With regards to the Copernicus space component two satellites are significant for the projects at the BKG. One is the satellite with a passive recording system, named Sentinel-2 and the other one is the satellite with an active recording system, named Sentinel-1 (Figure 1).

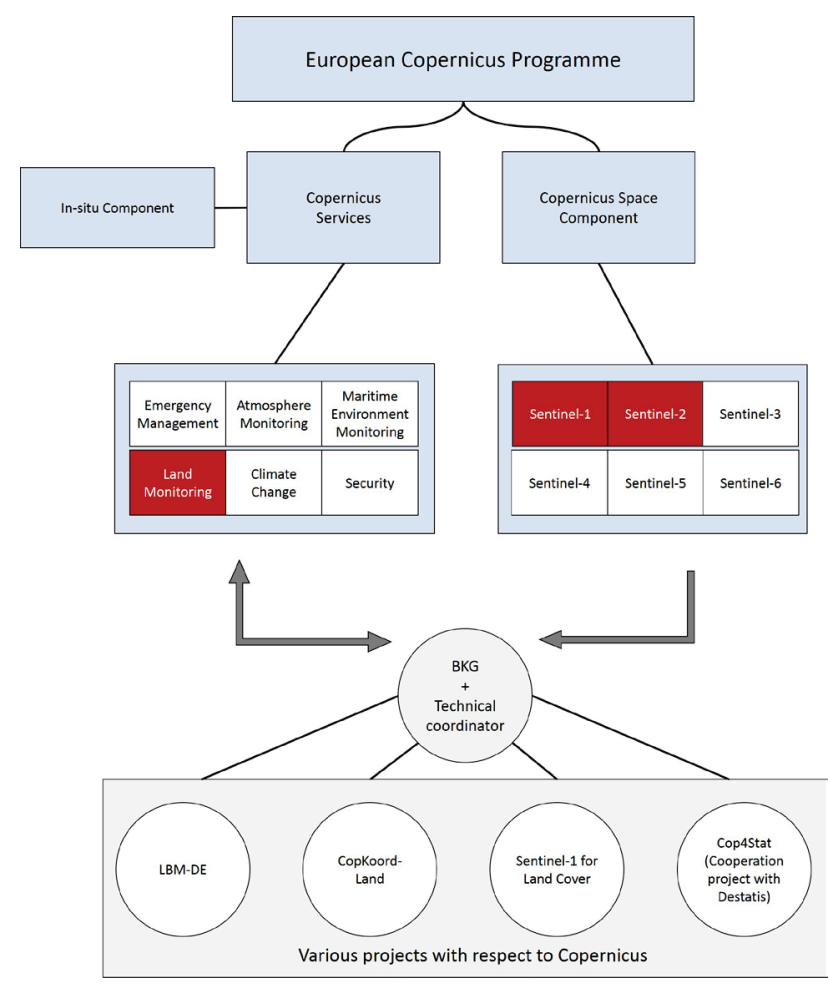

Figure 1: Simplified overview of the Copernicus programme (http://www.copernicus.eu/) showing the interaction and the current efforts at the BKG 
In terms of land monitoring with remote sensing datasets and especially with the aim to assist the Copernicus programme, different categories of projects exist at the BKG; for example i) projects that use Copernicus data and ii) projects which produce Copernicus data. The first type of projects use the different Sentinel datasets and Copernicus services to create their own individual land monitoring products. The second type is the most important project, namely the Digital Land Cover Model (LBM-DE), a national product of Germany, which contains separate information for LC and LU. More importantly, the LBM-DE product is used to create the European Corine Land Cover (CLC) classification products.

\subsection{Digital Land Cover Model for Germany: LBM-DE}

The Corine project with the CLC nomenclature has been widely used in the last decades (since the $90^{\text {th }}$ last century) as a European description of the environment and for European land monitoring. The CLC, with its hierarchical classification system, has a total of 44 classes for LC and LU information, which are not separated from each other (LC \& LU information are merged together in CLC). However, not all classes are relevant for the German environment and thus, the CLC for Germany consists of 37 classes. The CLC dataset is based on satellite image analysis and summarises the detected land cover with objects sizes greater than 25 ha in a representative mapping scale of 1:100.000 in Europe (European Environment Agency, 2000). Moreover, it is a pan-European thematic product that harmonises and unifies the land monitoring programme for Europe every six years (currently) with pre-defined specifications. These specifications have the same definitions over a long period of time and it is mandatory for all involved countries to follow them. 39 countries successfully submitted the national CLC in 2012 (land.copernicus.eu) after which this product is integrated in the Copernicus land monitoring service. To assist that European demand for CLC at a national level, the project Digital Land Cover Model for Germany (LBM-DE) was founded in 2006-2007 as a feasibility study (Arnold, 2009). In contrast to the CLC project, the LBM-DE has a three years cycle that is adapted to the CLC cycle. However, in 2009 the LBM-DE was created by using the CLC nomenclature. In 2012, the LBM-DE used a different nomenclature in comparison to the CLC classification. For this, classes from the ATKIS (Amtliches Topographisch - Kartographisches Informationssystem; Authoritative Topographic-Cartographic Information System) dataset were summarised and an independent product was produced. Nevertheless, the CLC2012 can be derived with the classification system of the LBM-DE, which separates LC and LU. In comparison to the CLC classes, the LBM-DE2012 has 36 LC and 15 LU classes with minimum 1 ha object sizes.

Therefore, the motivation to establish this new national dataset, LBM-DE, was on one hand, to support the CLC classification, but more importantly, to fulfil the national request of higher spatial and temporal resolution land cover products that separate LC and LU data (Hovenbitzer et al., 2014). Furthermore, the Copernicus services are supported by the LBM-DE and the LBM-DE presents an important contribution to the idea of a European Copernicus programme. Lastly, the projects described hereafter are strongly dependent on the LBM-DE.

\subsection{CopKoordLand}

CopKoordLand is a project that is responsible for assisting the technical coordination of the Copernicus land monitoring service in Germany, and is managed by the Federal Agency for Cartography and Geodesy. This project has two main objectives, a scientific one and the assistance of the technical coordination with respect to user uptake, communication with the European partners and public relations. In the scientific part, the combination of the Copernicus HRL with LBM-DE is evaluated. For instance, the derivation of the HRL from LBMDE2012 by a semantic transformation is investigated. Based on our analysis, we observed that this approach only works for the HRL permanent water bodies (Figure 2), and that in all other HRL, the definitions of the data content differs too much. As the LBM-DE2015 will have slightly different definition of classes, the evaluation will be repeated in the next years after the completion of LBM-DE2015.

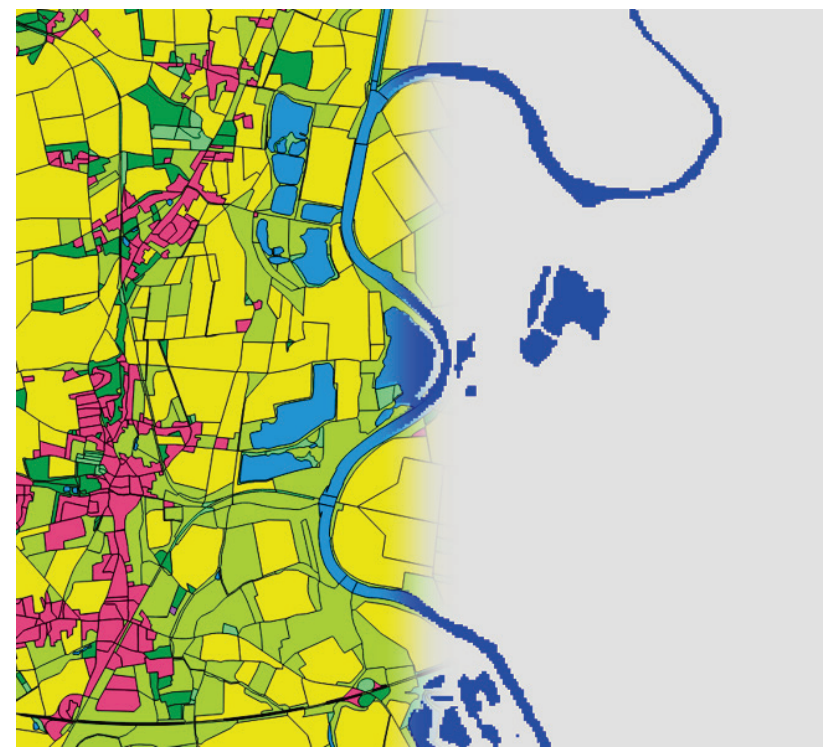

Figure 2: Derivation of the HRL permanent water bodies from LBM-DE

\subsection{Sentinel-1 for land cover classification}

The Sentinel-1 mission provides datasets by using Synthetic Aperture Radar (SAR) technology in the C-band frequency. A C-band SAR sensor actively transmits a pulse with a wavelength between 3.75 to $7.5 \mathrm{~cm}$ in the electromagnetic spectrum. The geometric resolution of the produced dataset is 5 to $20 \mathrm{~m}$ and the satellite has a repeat cycle of 12 days (every 3-4 days for Germany) (www.esa.int). The advantages of using Sentinel-1 datasets are: i) high resolution in horizontal geometry, ii) periodic imaging of the same target and iii) independence to daylight and weather conditions.

For this project, the Sentinel-1 data acquired in Interferometric Wide Swath mode (IW) and Ground Range Detected (GRD) datasets are used as they provide information about the backscattered signal in the imaged scene. The variation of the backscattered signal within a detected image depends on the physical properties of the targets such as geometry or roughness, the electric properties like dielectric composition or permittivity as well as the incidence angle of the sensor during imaging. The resulting images represent the backscatter of the different targets in the investigated areas (in the footprint of the detected area) as a varying greyscale distribution (Figure 3a). Consequently, a backscatter image gives information of target properties, such as soil moisture, soil surface roughness and the vegetation structure. Therefore, these images and the properties visible in them could be used for the separation of different 
objects in an image, and hence, the separation of different land cover classes.

Moreover, the Sentinel-1 IW images have different polarisation (dual polarisation $\mathrm{VV}$ and $\mathrm{VH}$ ) which allows for a polarimetric analysis of the imaged scene (Figure $3 b$ ).
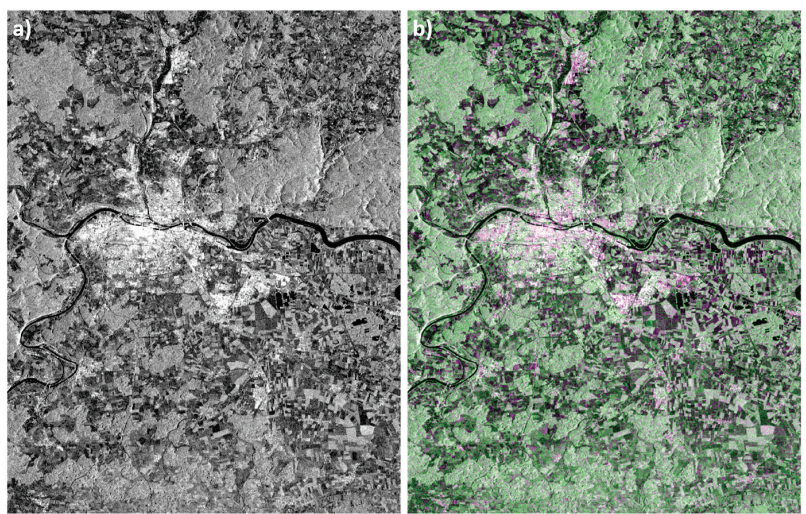

Figure 3: An example of the use of the Sentinel-1 dataset. a) VV polarisation image from 20. July 2015. b) RGB composite from $\mathrm{VV}, \mathrm{VH}$ and $\mathrm{VV}$ from the same area and day.

Our aim at the BKG is to use the Sentinel-1 datasets for extracting major land cover classes and to verify if a SAR based classification technique could provide land cover classes consistent with the LBM-DE classes. For this, the land cover classes in level 1 from the LBM-DE are used for training the SAR classifier and for validating the classification results. For a more detailed description of this project and a summary of the preliminary results, a separate publication can be found in this ISPRS archives (Suresh et al., 2016).

\subsection{Cop4Stat_2015plus}

In the context of land monitoring and the Copernicus programme, a cooperation project COP4STAT 2015plus was planned and proposed in 2014, and implemented in the second quarter of 2015. COP4STAT 2015plus stands for COPernicus for (4) STATistics and is a cooperation project between the Federal Statistical Office (Statistisches Bundesamt, DESTATIS project lead) and the Federal Agency for Cartography and Geodesy (Bundesamt für Kartographie und Geodäsie, BKG). The project aims at assessing the possibilities of the use of Copernicus remote sensing products for statistical purposes regarding information on $\mathrm{LC}$ and $\mathrm{LU}$.

The specific requirements for statistical information on $\mathrm{LC}$ and LU come from the European classification system of LUCAS (Land Use / Cover Area Frame Survey) as used by the European Statistical Agency 'Eurostat'. LUCAS is a point-based sample field survey, which is executed every three years (Eurostat, 2013). So far, it is executed throughout the EU following a harmonised set of specifications for field data collection and is independent from the EU Member States' national statistical surveys. Due to different methods and updating approaches between Eurostat's LC / LU area estimates (based on LUCAS point sample) and the national statistical data sources, the results on the European and national levels are often not entirely comparable. Therefore, in the future, Eurostat plans for a stronger integration of national data sources in the evaluation of LUCAS area estimates, by asking the EU Member States to contribute with data on LC and LU in the form of statistical tables. Through this approach, the LUCAS nomenclature becomes a target nomenclature also at the national level.

The LUCAS classification system provides and allows for the description of land from both the LC and the LU perspectives. This classification system has a total of 73 classes for LC (without LUCAS forest types) and 36 classes for LU. For the LC, there exist 8 classes in level 1, 28 classes in level 2 and 60 classes in level 3. For the LU, 4 classes in level 1, 16 classes in level 2 and 26 classes in level 3 are described (Eurostat LUCAS 2015 Technical document C3 - Classification).

Most existing national land surveying classification systems or feature type catalogues consist of mixed nomenclatures, meaning that they are composed of classes that either contain information on LC or on LU. As a consequence, it is not possible to derive information on both LC and LU from national sources that fully match Eurostat's LUCAS nomenclature, because in many cases either the LC or the LU part of information is missing.

As the outcome of a pilot study (2013-2014) to derive LC and LU data from existing national data sources according to the LUCAS classes, it was found that no national dataset in Germany - neither the areal statistics nor topographic data - can be directly used as a stand-alone source for the derivation of all required target classes, even on the generic basis of level 1 LUCAS classes (Arnold, 2015). To close this gap, remote sensing data shall now be assessed as an additional source of information within the Cop4Stat-Project. However, “... remote sensing is difficult to use for the LUCAS classification at the 3rd level of detail." (Eurostat - LUCAS 2015 Technical document C3 - Classification). To find solutions for these challenges is one of the primary goals in this cooperation project. The first step is to take the results of the above mentioned LUCAS pilot study for analysing the semantic matching of definitions between national areal statistical classes and LUCAS classes. Since the usage of only a single national dataset is insufficient, several datasets are investigated in order to calculate the area sizes of all remaining LUCAS classes. Currently, the areal statistical results on national level are based on 17 classes from ALB (Automatisiertes Liegenschaftsbuch; Automated Real Estate Book) which are provided as the minimum common publication standard by the land surveying and cadastral authorities of the Federal States (Destatis, 2013). However, in the near future, the ALKIS (Amtliches Liegenschaftskataster-Informationssystem; Authoritative Real Estate Cadastre Information System) data will be used for the areal statistical calculation, then with an extended number of 28 classes (Destatis, 2014).

After the semantic transformation between the national and European class requirements, the next step is investigating the possibilities to extract additional information from Copernicus remote sensing data and derived products. In this case, the focus lies on the national statistical classes, which cannot directly be transformed into the LUCAS classes, and to find out where additional information on LC or LU is required to support the LUCAS nomenclature. Consequently, another aim of the project is to identify reproducible and consistent methods to derive LC and LU information from remote sensing and national data which can be analysed for the purpose of areal statistical calculations.

Multispectral satellite images from Sentinel-2 will be used as input data for the LC analysis. As additional data sources, other existing national datasets (the German national land cover model LBM-DE and/or ATKIS) will be integrated in the work process for acquiring LU information. This approach will be tested in selected areas and a comparison will be made between the calculated statistical results from remote sensing data and 
the LC or LU area sizes from official areal statistics (based on cadastral data).

To summarise, the goal of the project Cop4Stat_2015plus is to extract information on landscape from remote sensing data, relevant for national areal statistical calculations. This will be accomplished by the completion of three main correlated tasks: i) analysing the different nomenclature used by the existing national and international classification systems, ii) image processing of remote sensing datasets for land cover and analysis of national datasets for the land use classification, iii) calculation of areal statistics based on these two results. The results can be used for supporting the national and the European statistical information as it aims at covering both their requirements. Moreover, the representation of the results in a spatial view with spatial distribution is possible and therefore changes in LC and LU can be monitored and detected.

\section{SUMMARY AND OUTLOOK}

This paper illustrates how Copernicus data can be used in the daily practice of a national mapping agency in terms for applications like land monitoring. The BKG integrates the Copernicus programme into its present activities as follows: i) by using the Copernicus products and data, ii) by coordinating the Copernicus land monitoring services for Germany, as well as iii) by producing Copernicus related products. The four projects described here, show the potential of using the Copernicus programme at a Federal agency, such as ours, for different applications related to land cover classification and monitoring. While the three projects, Sentinel-1 for Land cover classification, Cop4Stat and CopKoordLand use Copernicus provided products, the LBM-DE created at the BKG, is integrated by the Copernicus programme, to create the CLC.

However, it is also evident that a greater requirement of expertise, infrastructure and practical applications are needed to comprehend, support and satisfy the possibilities of the Copernicus programme. These can only be fulfilled by the initiation of prospective projects and this must be emphasised in the near future to assist the Copernicus services within the different thematic topics. This not only concerns the classical geo-topographical subjects, but also other research topics (such as geology, hydrology, traffic management, climate, atmosphere, natural hazards, regional and local planning, etc.) where spatial data and information are essential. The national technical coordinators of the different thematic topics are responsible for addressing and solving the challenges encountered within each project. By working together to solve the various problems, a common structure for the entire national Copernicus programme can be established.

Finally, to exploit the full potential of the Copernicus programme, interdisciplinary and international projects should also be encouraged and continued in the future.

\section{ACKNOWLEDGEMENTS}

The team of the LBM-DE at the BKG had a big influence on the other projects and so we would like to extend our greatest gratitude to Christine Wende, Friederike Emig, Katja Happe, Gabriele Runkel and Viola Brandel.

We would also like to thank the cooperation partner DESTATIS (project leader) of the Cop4Stat project and, specifically, Sarah Kleine, Stephan Arnold and Hermann Seewald for their fruitful discussions during this project.

\section{REFERENCES}

Arnold, S., 2009. Digital Landscape Model DLM-DE Deriving land cover information by integration of topographic reference data with remote sensing data, Proceedings ISPRS Workshop Hannover 2009. Volume XXXVIII-1-4-7/W5. http://www.isprs.org/proceedings/XXXVIII/1_4_7W5/paper/Arnold-167.pdf (March 2016).

Arnold, S., 2015. Bereitstellung harmonisierter Landnutzungsund Landbedeckungsstatistiken - Pilotstudie zur Unterstützung der europäischen LUCAS-Erhebung. Statistisches Bundesamt, WISTA, 2, 2015, 67-79.

Destatis, 2013. Flächenerhebung nach Art der tatsächlichen Nutzung. Qualitätsbericht. Statistisches Bundesamt, Wiesbaden. www.destatis.de

Destatis, 2014. Land- und Forstwirtschaft, Fischerei. Bodenflächen nach Art der tatsächlichen Nutzung. Fachserie 3, Reihe 5.1, Statistisches Bundesamt, Wiesbaden 2015. www.destatis.de

European Environment Agency, 2000. Corine land cover technical guide - Addendum 2000. Technical report No. 40. Copenhagen $2000 . \quad \mathrm{http} / / /$ land.copernicus.eu/usercorner/technical-library (March 2016).

Eurostat, 2013. LUCAS - The EU's land use and land cover survey. Compact Guide. Luxemburg 2013. ec.europa.eu/eurostat (March 2016).

Eurostat technical document, E4.LUCAS (ESTAT), Regional statistics and Geographic Information. LUCAS 2015 (Land Use / Cover Area Frame Survey), Technical reference document C3 Classification (land cover \& land use).

Hovenbitzer, M., Emig, F., Wende, C., Arnold, S., Bock, M. \& Feigenspan, S., 2014. Digital Land Cover Model for Germany DLM-DE. In: Manakos, I. \& Braun, M. (Hg.) Land Use and Land Cover Mapping in Europe: Practices \& Trends, Remote Sensing and Digital Image Processing 18, Springer Verlag, 255272 .

Jetzek, T., 2013. The value of open data government data. Perspektiv Nr. $23 \quad-2013,47-56$, http://journals.aau.dk/index.php/gfp/article/download/489/400 (March 2015).

Suresh, G., Gehrke, R., Wiatr, T., Hovenbitzer, M., 2016. Synthetic aperture radar (SAR) based classifiers for land applications in Germany. ISPRS Congress Archives. Prague.

http://www.copernicus.eu/ (February 2016).

https://scihub.copernicus.eu/ (February 2016).

land.copernicus.eu (February 2016).

www.esa.int (February 2016).

SNAP - ESA Sentinel Application Platform v2.0.2, http://step.esa.int (February 2016). 